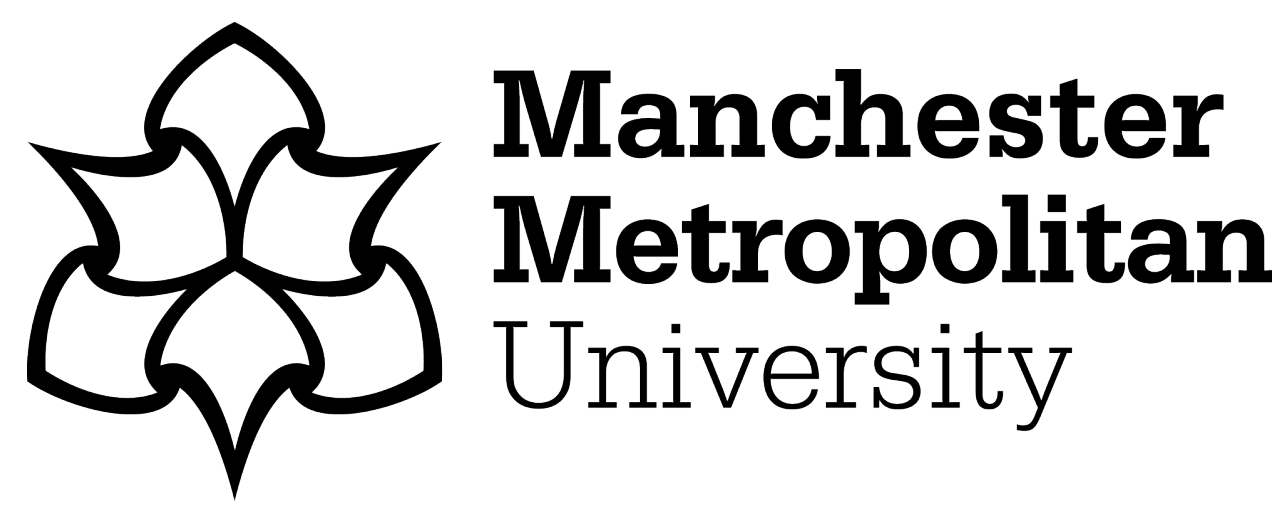

Scarth, Morgan, Havnes, Ingrid A, Jørstad, Marie L, McVeigh, Jim ORCID logoORCID: https://orcid.org/0000-0001-5319-6885, Van Hout, Marie Claire, Westlye, Lars T, Torgersen, Svenn and Bjørnebekk, Astrid (2022) Severity of anabolic steroid dependence, executive function, and personality traits in substance use disorder patients in Norway. Drug and Alcohol Dependence, 231. p. 109275. ISSN 0376-8716

Downloaded from: https://e-space.mmu.ac.uk/628990/

Version: Published Version

Publisher: Elsevier

DOI: https://doi.org/10.1016/j.drugalcdep.2022.109275

Usage rights: Creative Commons: Attribution 4.0

Please cite the published version 


\title{
Severity of anabolic steroid dependence, executive function, and personality traits in substance use disorder patients in Norway
}

\author{
Morgan Scarth ${ }^{\mathrm{a}, \mathrm{b}, *}$, Ingrid A. Havnes ${ }^{\mathrm{c}}$, Marie L. Jørstad ${ }^{\mathrm{c}}$, Jim McVeigh ${ }^{\mathrm{d}}$, \\ Marie Claire Van Hout ${ }^{\mathrm{e}}$, Lars T. Westlye ${ }^{\mathrm{b}, \mathrm{f}, \mathrm{g}}$, Svenn Torgersen ${ }^{\mathrm{b}}$, Astrid Bjørnebekk ${ }^{\mathrm{a}}$ \\ a Anabolic Androgenic Steroid Research Group, Section for Clinical Addiction Research, Division of Mental Health and Addiction, Oslo University Hospital, Oslo, Norway \\ ${ }^{\mathrm{b}}$ Department of Psychology, University of Oslo, Oslo, Norway \\ ${ }^{\mathrm{c}}$ Anabolic Androgenic Steroid Research Group, National Advisory Unit on SUD Treatment, Division of Mental Health and Addiction, Oslo University Hospital, Oslo, \\ Norway \\ ${ }^{\mathrm{d}}$ Substance Use \& Associated Behaviours, Department of Sociology, Manchester Metropolitan University, Manchester, UK \\ ${ }^{\mathrm{e}}$ Public Health Institute, Faculty of Health, Liverpool John Moore's University, Liverpool, UK \\ ${ }_{\mathrm{f}}^{\mathrm{f}}$ KG Jebsen Center for Neurodevelopmental Disorders, University of Oslo, Oslo, Norway \\ ${ }^{g}$ NORMENT, Division of Mental Health and Addiction, Oslo University Hospital \& Institute of Clinical Medicine, University of Oslo, Norway, University of Oslo, Oslo, \\ Norway
}

\section{A R T I C L E I N F O}

\section{Keywords:}

Anabolic androgenic steroids

Personality

Executive function

Latent class analysis

Substance use disorder

\begin{abstract}
A B S T R A C T
Introduction: Anabolic androgenic steroids (AAS), including testosterone and synthetic derivatives, are typically used to increase muscle mass. Many users develop a dependence on these substances, contributing to worsened physical and mental health outcomes. Aspects of personality and executive dysfunction may represent underlying vulnerabilities for developing dependence.

Objective: To identify levels of AAS dependence within substance use disorder (SUD) treatment patients and assess the relationship between dependence severity and personality traits and executive function (EF).

Methods: Data were collected from patients at 38 SUD treatment facilities in Norway. Questionnaires were completed for measures of personality and EF. Measures of symptoms of AAS dependence were used in latent class analysis to identify sub-groups of patients, which were evaluated for association with EF and personality traits, and compared with a group of non-AAS using SUD patients.

Results: Three classes were identified; largely reflecting low, moderate, and high symptoms of dependence. Multinomial regression analyses indicated that moderate and high symptoms were associated with several measures of EF and personality traits, particularly self-monitoring, antagonism, disinhibition, and rigid perfectionism while users with low symptoms exhibited higher capacities for emotional control and shift, and lower negative affectivity, relative to non-AAS using SUD patients. Backward stepwise regressions indicated antagonism, and decreased self-monitoring as key personality and cognitive characteristics of SUD patients with severe AAS dependence.

Conclusion: Our findings indicate that specific executive dysfunctions and personality features, particularly those associated with poor emotional control, reduced empathy, and impulsivity are associated with more severe AAS dependence in the SUD population.
\end{abstract}

\section{Introduction}

Anabolic-androgenic steroids (AAS) include testosterone and synthetic derivatives, which are primarily used by athletes and weightlifters to increase muscle mass. Supra-physiological doses of testosterone may lead to numerous adverse health consequences, including increased risk of somatic and psychiatric problems such as cardiac conditions, anxiety, and aggression (Chegeni et al., 2021; Horwitz et al., 2019; Piacentino et al., 2015; Pope et al., 2014; van Amsterdam et al., 2010). Approximately one-third of users develop a

\footnotetext{
* Correspondence to: Anabolic Androgenic Steroid Research Group, Section for Clinical Addiction Research, Division of Mental Health and Addiction, Oslo University Hospital, Postbox 4959 Nydalen, 0424 Oslo, Norway.

E-mail address: morsca@ous-hf.no (M. Scarth).
} 
dependence on these substances, and subsequently increase the risk of adverse side-effects (Hauger et al., 2021; Ip et al., 2012; Kanayama et al., 2009b, 2010). Additionally, AAS use is more prevalent among those with a substance use disorder (SUD) compared to the general population (Dodge and Hoagland, 2011; Havnes et al., 2020b; Sagoe et al., 2015; Salinas et al., 2019). Relative to non-AAS substance users, those who use AAS report a younger substance use debut age and a greater number of substances used, suggesting more severe substance use within this group (Havnes et al., 2020a, 2020b).

AAS dependence can be characterized as continuing use despite negative physical and psychological outcomes including withdrawal symptoms and impaired psychosocial functioning (Kanayama et al., 2009a, 2009b), and is likely driven by a combination of psychological factors including body image and appearance concerns, personality traits, and physiological problems, primarily hypogonadism (Griffiths et al., 2018). SUD patients using AAS exhibit substantial variability in the severity and nature of their use, which may be partly explained by cognitive and personality characteristics. In order to characterize relevant individual differences in AAS dependence within this group, a dimensional approach may be beneficial. This approach reflects the recent changes towards more individualized care in psychiatry, as demonstrated by the Diagnostic and Statistical Manual, 5th edition, and the Eleventh Revision of the International Classification of Diseases which include levels of severity within SUD (American Psychiatric Association, 2013; World Health Organization, 2019).

Underlying risks for more severe dependence may include cognitive difficulties and certain personality traits. Executive functions (EFs) include higher-level cognitive skills required for coordinating and controlling behaviors and other cognitive abilities, such as emotional control and impulse inhibition. Executive dysfunctions have been associated with several disorders, including SUD (Diamond, 2013). Patients with SUD have demonstrated difficulties with inhibition, self-monitoring, and working memory (Wilens et al., 2017). In previous studies, individuals with poly-substance use disorder performed significantly worse than healthy controls on several aspects of EF, with the strongest effect for emotional control (Hagen et al., 2016), which may be applicable to AAS users in SUD treatment. AAS users have also exhibited higher levels of impulsivity (Garcia-Argibay, 2019), and may have deficits in aspects of memory and cognition (Bjornebekk et al., 2019; Heffernan et al., 2015; Kanayama et al., 2013). Furthermore, our research group has previously reported deficits in working memory, mental flexibility, problem-solving, and inhibition in those with AAS dependence compared to non-AAS using weight-lifting controls (Hauger et al., 2020). However it is difficult to determine to which degree these group differences reflect the consequences of AAS use, preexisting risk factors or a combination.

Personality pathology, including maladaptive patterns of behavior, cognition, and emotion, is common among both AAS users and SUD patients. Previous studies suggest that AAS users may exhibit high levels of neuroticism and impulsivity (Garcia-Argibay, 2019), while SUD appears to be related to disinhibition (Kotov et al., 2010). In addition, compared to non-using weightlifters, AAS users tend to display characteristics of cluster B personality disorders: narcissistic, antisocial, histrionic, and borderline (Hauger et al., 2021; Perry et al., 2003; Porcerelli and Sandler, 1995). The unpredictable and overly emotional nature of these personality facets contribute to both intra- and inter-personal challenges. In addition, SUD frequently co-occurs with borderline personality disorder (Trull et al., 2018), a disorder that is marked by emotional dysregulation (Snow et al., 2020), suggesting a connection with certain EFs that may be present in both AAS users and SUD patients.

In addition to the potential risk of psychiatric and somatic health problems, those who develop AAS dependence may exhibit more impulsive and aggressive behavior, putting those around them at risk, making it critical to understand which characteristics contribute to AAS dependence. To this end, in this study we aimed to identify latent classes of AAS dependence within the SUD population, and assess the association of these classes with measures of personality and EF.

\section{Methods}

Data were drawn from our cross-sectional study Anabolic steroid use among patients in substance use disorder treatment in Norway, which includes 38 SUD treatment institutions/facilities in Norway. The management of SUD treatment facilities were contacted and informed about the study, and data collection was largely organized by the research group. A more detailed description of the recruitment procedures and characteristics of SUD treatment in Norway is available (Havnes et al., 2020b).

\subsection{Participants}

Participants were included if they were currently active in in- or outpatient SUD treatment, were at least 18 years of age, and were able to read Norwegian language. The majority of participants were inpatients (80\%), and all participants were in SUD treatment for a variety of substances not including AAS. Written informed consent was obtained. The original sample contained 565 participants. Those who were determined to have unreliable responses, for example due to skipping several relevant items $(n=14)$ or missing information for AAS use $(n=10)$ were removed. AAS users that were missing dependence questionnaire data $(\mathrm{n}=20)$ were not included in the analyses. The final sample comprised of 521 patients (389 men). Within this sample, 136 (26\%) reported lifetime use of AAS. Of those that used AAS, 130 (96\%) were male.

\subsection{Questionnaires}

\subsubsection{Demographics and psychoactive substance use}

Background and health information were recorded, including gender, age, and level of education. Previous or current prescribed medication was registered, and weight and height were used to calculate body mass index (BMI). Psychoactive substance use was evaluated using selected items from the European Addiction Severity Index, adapted for this study (Blacken et al., 2010). These items evaluated characteristics of substance use behavior including age at onset of substance use, and age at first SUD diagnosis, preferred substance(s), and most frequently used substances. Psychoactive substances included alcohol, stimulants, sedatives, opioids, and cannabis. To assess the prevalence of polysubstance use, participants were asked how many substances they used per week, which was categorized as $1,2-3$, and 4 or more substances.

\subsubsection{AAS use and dependency}

Information regarding AAS use was recorded including age at onset of use, most commonly used types of AAS, and frequency of use. Participants were considered to use a type of AAS if they reported a compound as one of their three most frequently used AAS. Duration of AAS use was reported in years and months; participants who reported taking AAS orally or by injection one or a few times were recorded with one month of use. AAS dependency was measured using a Norwegian translation of the DSM-IV-adapted questionnaire (Ip et al., 2012), which is based on the SCID-II for substance dependence, with adaptations for AAS dependence. Participants reported the presence or absence of nine symptoms of AAS dependence. Of these, five symptoms (tolerance, larger amounts, withdrawal, use to relieve withdrawal, use despite problems) were selected for analysis based on theoretical relevance and statistical contribution to the latent class model (Table S1). For comparison, dependence was also evaluated with the standard cut-off score: the presence of three or more symptoms of AAS dependence.

\subsubsection{Personality}

Participants completed a 34-item questionnaire using nine items from the Severity Indices of Personality Problems (SIPP-118) (Verheul 
et al., 2008) and 25 items comprising the Personality Inventory for DSM-5 Brief Form (PID-5-BF) (Krueger et al., 2012). Responses ranged from "very wrong or often wrong" (0), "sometimes or a little wrong" (1), "sometimes or a little true" (2), to "very true or often true" (3). The mean of items from each of the following scales was computed: from the PID-5 negative affectivity, detachment, antagonism, disinhibition, and psychoticism, and rigid perfectionism, from the SIPP-118 identity integration and relational capacities.

\subsubsection{Executive function}

Executive function was assessed using a short version of the Behavior Rating Inventory of Executive Function-Adult (BRIEF-A) (Roth and Gioia, 2005), which originally consists of 75 items measured on a three-point response scale; "never" (1), "sometimes" (2), and "often" (3). For this study, the three questions with the highest correlation coefficient of each subscale were used. The mean of items in each of the nine non-overlapping subscales was computed: inhibit, working memory, emotional control, shift, self-monitor, task monitor, organization of materials, initiate, and plan/organize. In addition, the subscales can be summed into metacognition (initiate, plan/organize, working memory, organization of materials, task monitor) and behavioral regulation (inhibit, shift, self-monitor, emotional control) indices.

\subsection{Statistical analysis}

All statistical analyses were done in $\mathrm{R}$ version 4.0.3 ( $\mathrm{R}$ Core Team, 2018). Latent class analysis was used to identify subgroups of AAS dependency among all those who reported AAS use, based on responses to the dependency questionnaire items using the R package poLCA (Linzer and Lewis, 2011). Items were assessed for added value in the latent class model, and five items were included in the final model (Table S1). LCA models with 1-6 classes were computed and compared using goodness-of-fit statistics including Bayesian Information Criteria (BIC), sample size adjusted BIC, and entropy to determine the optimal model. Entropy, which measures how well the classes are separated by the model, is generally considered to be acceptable at 0.8 or higher (Tein et al., 2013). Subsequent analyses utilized one-way ANOVA and Tukey HSD post-hoc test to compare differences in EF and personality measures among the various dependency classes.

Multinomial logistic regression was used to assess the relationship between most likely class membership, and each measure of personality and EF. Odds ratios with a 95\% confidence interval were calculated using the non-AAS using participants as a reference category. The ANOVA and multinomial regression analyses were repeated excluding females to account for the low proportion of AAS-using females. The relationship among components of personality and EF were assessed with Kendall's rank correlation (tau), as a non-parametric alternative to Pearson's correlation coefficient. Backwards stepwise regression with a threshold of $\mathrm{p}<0.1$ were used for both personality and EF measures independently to identify the most prominent characteristics of dependence severity. Multicollinearity was assessed using variance inflation factors.

\subsection{Ethics and data privacy}

This study was approved by the data protection officer for research at the Oslo University Hospital (2016/1119), and all research was executed in accordance with the Declaration of Helsinki. All participants received oral and written information regarding the study, and written formal consent was provided by all participants.

\section{Results}

The total sample consisted of 529 participants (74.7\% males) with a mean age of 37.8 years. The average debut age of any substance was 14.5 years, with $37.3 \%$ of the sample reporting alcohol as their most used substance. The demographic and substance use characteristics of the sample can be found in Table 1.

\subsection{Latent class analysis}

A three-class model was chosen as the optimal choice, based on the relatively high entropy, and relatively low adjusted BIC. Complete fit statistics can be found in Table $\mathrm{S} 2$. The classes can be summarized as low dependence symptoms $(\mathrm{n}=71)$, moderate dependence symptoms $(\mathrm{n}=$ 38 ), and high dependence symptoms $(\mathrm{n}=27)$, in addition to SUD patients who had never used AAS $(\mathrm{n}=389)$. Low dependence symptoms users reported very few symptoms of AAS dependence; $35.2 \%$ reported withdrawal, and $4.2 \%$ of these users would qualify as a dependent using a cut-off score. All users classified with moderate dependence symptoms reported taking larger amounts of AAS longer than intended (100\%), and many reported symptoms of tolerance (50\%) and withdrawal (60.5\%), though few reported using AAS to relieve withdrawal symptoms $(10.5 \%)$, or continued use despite side effects $(21.1 \%) ; 73 \%$ of users in this group would qualify as dependent using cut-off scores. The high dependence symptoms class can be characterized by a high prevalence of all symptoms, notably using AAS to relieve symptoms of withdrawal (100\%), and all members would be considered dependent by dichotomous measures (Fig. 1 and Table S3).

Those who did not use AAS were older ( 39.48 years \pm 12.34 ) than all classes of AAS users (low: $34.31 \pm 7.37$, moderate: $30.26 \pm 5.49$, high: $33.81 \pm 7.42)$. The moderate and high dependence symptoms groups began using substances earlier (moderate: 13.01 years \pm 2.11 , high: $13.44 \pm 1.99)$ than those with low dependence symptoms $(14.29 \pm 2.95)$ and non-AAS users $(14.81 \pm 4.14)$. High dependence symptoms users had used AAS for a longer duration (median 36 months) than the moderate (17 months) and low (4 months) symptoms classes. All members of the high symptoms class were male, and males represented $92.1 \%$ of the moderate symptoms class, $95.8 \%$ of the low symptoms class, and $67.3 \%$ of those that did not use AAS. Additionally, $37 \%$ of the high and $28.9 \%$ of the moderate symptoms classes reported stimulants as the most frequently used substance, more than both the low symptoms (23.5\%) and non-AAS user (14.1\%) groups. More than half of total sample reported polysubstance use, with higher prevalence in all classes of AAS users (between $87 \%$ and $89 \%$ ) than non-AAS users (55.5\%). The most commonly used AAS in all groups was testosterone. The high symptoms class used trenbolone more than the low or moderate symptoms classes $(48.1 \%)$, while the moderate symptoms class had the greatest use of nandrolone (34.2\%). Full demographic and substance use descriptive statistics can be found in Table 1 . Due to the low proportion of females using AAS in this population, only males were included in the subsequent analyses, with results using the whole study sample presented in the Supplemental Materials.

\subsection{Executive function}

The high dependence symptoms class demonstrated greater executive dysfunction compared to non-AAS users on measures of emotional control, self-monitoring, and the sum score behavioral regulation. Additionally, this class demonstrated greater deficits in shift, emotional control, behavioral regulation and self-monitoring compared to those with low dependence symptoms. The moderate symptoms class demonstrated poorer self-monitoring than the low symptoms class and non-AAS users (Table 2, Fig. 2, females included Table S4).

Regression results indicated a negative association of shift (OR 0.53; 95\% CI: 0.30-0.93) and emotional control (OR 0.48; 95\% CI: 0.25-0.91) for the low symptoms class (i.e. poorer functioning on these measures was associated with lower probability of being in this class, compared to non-AAS users). Moderate symptoms class membership was associated with both impulse inhibition (OR 1.98; 95\% CI: 1.00-3.92) and selfmonitoring (OR 2.64; 95\% CI: 1.29-5.38), in addition to task monitoring (OR 2.30; 95\% CI: 1.08-4.92). High symptoms class membership 
Table 1

Demographic variables of sample by latent classes of dependence symptoms.

\begin{tabular}{|c|c|c|c|c|c|c|}
\hline & Overall & Non-AAS users & Low symptoms & Moderate symptoms & High symptoms & $\mathrm{p}$ \\
\hline $\mathrm{N}$ & 521 & 385 & 71 & 38 & 27 & \\
\hline Age (mean (SD)) & $37.82(11.56)$ & $39.48(12.34)$ & $34.31(7.37)$ & $30.26(5.49)$ & $33.81(7.42)$ & $<0.001^{\mathrm{A}, \mathrm{B}}$ \\
\hline Sex $=$ female $(\%)$ & $132(25.3)$ & $126(32.7)$ & $3(4.2)$ & $3(7.9)$ & $0(0.0)$ & $<0.001^{\mathrm{A}, \mathrm{B}, \mathrm{C}}$ \\
\hline Years of education (mean (SD) & $11.74(2.06)$ & $11.79(2.02)$ & $11.91(1.94)$ & $11.40(2.50)$ & $11.08(2.29)$ & 0.24 \\
\hline Debut age any drug & $14.54(3.82)$ & $14.81(4.14)$ & $14.29(2.95)$ & $13.01(2.11)$ & $13.44(1.99)$ & $0.016^{\mathrm{B}}$ \\
\hline Debut age AAS & $22.92(6.11)$ & NA & $24.89(6.39)$ & $20.75(4.43)$ & $20.78(5.80)$ & $<0.001^{\mathrm{D}, \mathrm{E}}$ \\
\hline Duration of use (months) (median [IQR]) & $12.00[3.00,24.00]$ & NA & $4.00[2.50,12.00]$ & $17.00[6.75,36.00]$ & $36.00[18.00,67.00]$ & $<0.001 * \mathrm{D}, \mathrm{E}$ \\
\hline Most frequently used substance (\%) & & & & & & $<0.001$ \\
\hline Alcohol & $192(37.3)$ & $174(45.5)$ & $11(16.2)$ & $5(13.2)$ & $2(7.4)$ & \\
\hline Stimulants & $91(17.7)$ & $54(14.1)$ & $16(23.5)$ & $11(28.9)$ & $10(37.0)$ & \\
\hline Sedatives & $26(5.0)$ & $16(4.2)$ & $5(7.4)$ & $4(10.5)$ & $1(3.7)$ & \\
\hline Opioids & $58(11.3)$ & $34(8.9)$ & $13(19.1)$ & $5(13.2)$ & $6(22.2)$ & \\
\hline Cannabis & $62(12.0)$ & $39(10.2)$ & $11(16.2)$ & $9(23.7)$ & $3(11.1)$ & \\
\hline Other & $12(2.3)$ & $7(1.8)$ & $3(4.4)$ & $0(0.0)$ & $2(7.4)$ & \\
\hline Number of substances used/week (\%) & & & & & & $<0.001$ \\
\hline 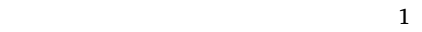 & $173(36.0)$ & $158(44.5)$ & $8(12.3)$ & $4(11.4)$ & $3(12.0)$ & \\
\hline $2-3$ & $154(32.1)$ & $110(31.0)$ & $27(41.5)$ & $11(31.4)$ & $6(24.0)$ & \\
\hline 4 or more & $153(31.9)$ & $87(24.5)$ & $30(46.2)$ & $20(57.1)$ & $16(64.0)$ & \\
\hline \multicolumn{7}{|l|}{ Type of AAS used $* *=n(\%)$} \\
\hline Testosterone & $68(13.1)$ & NA & $27(38.0)$ & $22(57.9)$ & $19(70.4)$ & $.008^{\mathrm{D}, \mathrm{E}}$ \\
\hline Nandrolone & $33(6.3)$ & NA & $13(18.3)$ & $13(34.2)$ & $7(25.9)$ & 0.178 \\
\hline Trenbolone & $34(6.5)$ & NA & $8(11.3)$ & $13(34.2)$ & $13(48.1)$ & $<0.001^{\mathrm{D}, \mathrm{E}}$ \\
\hline Dianabol & $25(4.8)$ & NA & $14(19.7)$ & $7(18.4)$ & $4(14.8)$ & 0.855 \\
\hline Stanozolol & $23(4.4)$ & NA & $11(15.5)$ & $6(15.8)$ & $6(22.2)$ & 0.713 \\
\hline
\end{tabular}

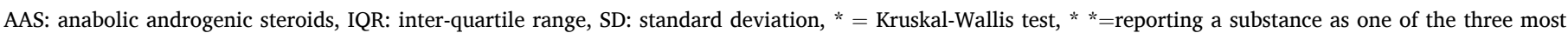

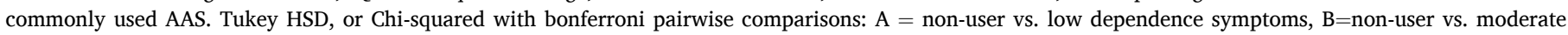

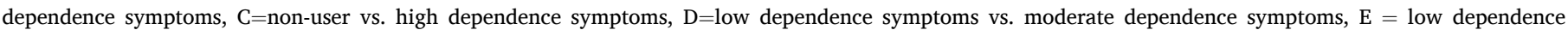
symptoms vs. high dependence symptoms, $\mathrm{F}=$ moderate dependence symptoms vs. high dependence symptoms
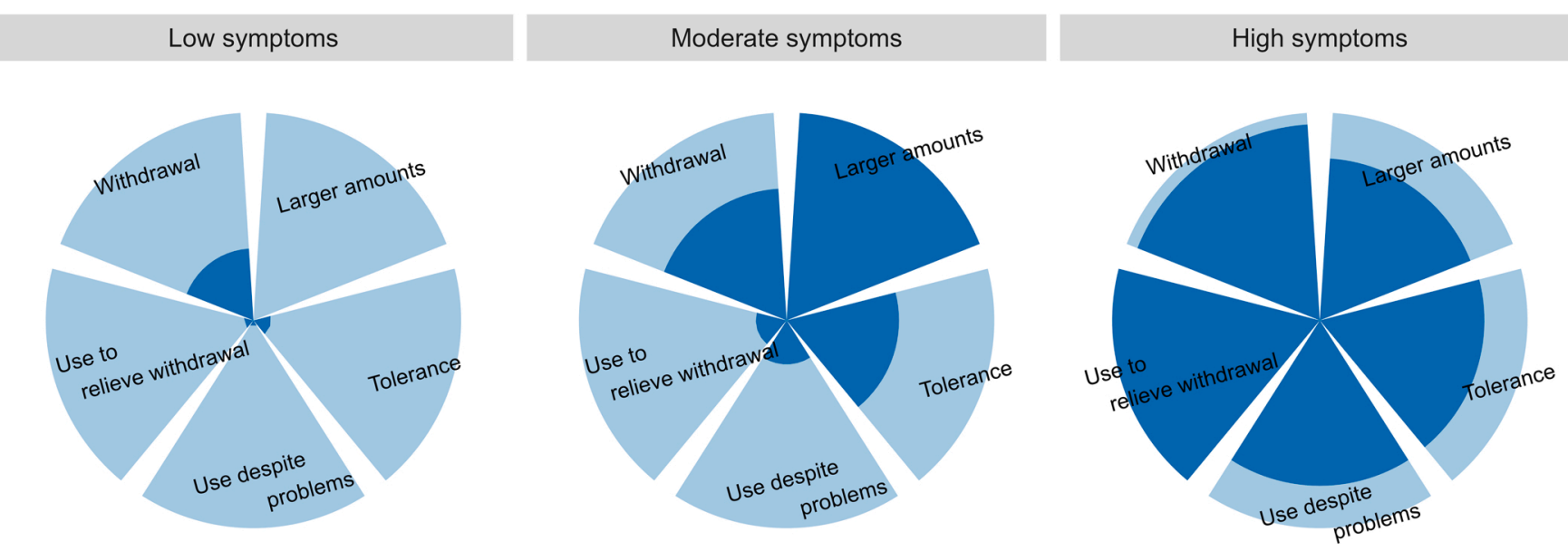

\section{Symptom present $\square$ Yes No}

Fig. 1. Dependence symptoms reported in each of the three identified latent classes (low, moderate, and high symptoms). Darker area indicates the proportion of participants reporting each dependence symptom in each latent class, lighter area indicates the proportion of participants not reporting each symptom.

was associated with self-monitoring (OR 3.32; 95\% CI: 1.49-7.40), emotional control (OR 3.31; 95\% CI: 1.33-8.21), and behavioral regulation (OR 1.53; 95\% CI: 1.16-2.01) (Table 3, females included Table S5).

Backward stepwise regression analysis included impulse inhibition, emotional control, and self-monitoring in the best fitting model. Impulse inhibition was positively associated with low dependence symptoms (OR 2.12; 95\% CI: 1.07-4.19). Emotional control was negatively associated with low (OR 0.35; 95\% CI: 0.15-0.81) symptoms. Selfmonitoring was positively associated with both moderate (OR 2.87; 95\% CI: 1.07-7.73) and high (OR 3.64; 95\% CI: 1.18-11.30) dependence symptoms (Table S6).

\subsection{Personality traits}

The high dependence symptoms class demonstrated higher negative affectivity compared to the low symptoms classes. On measures of antagonism, disinhibition, rigid perfectionism, identity integration, and relational capacities, the moderate and high symptoms classes differed very little. However, the moderate symptoms class demonstrated increased antagonism and disinhibition compared to non-users. The high symptoms class demonstrated increased levels of antagonism and 
Table 2

Descriptive statistics of personality domains and executive function, mean (standard deviation).

\begin{tabular}{|c|c|c|c|c|c|}
\hline & Non-AAS user & Low symptoms & Moderate symptoms & High symptoms & $\mathrm{p}$ \\
\hline $\mathrm{n}$ & 259 & 68 & 35 & 27 & \\
\hline \multicolumn{6}{|l|}{ Executive function } \\
\hline Inhibit & $2.07(0.54)$ & $2.11(0.52)$ & $2.27(0.55)$ & $2.28(0.59)$ & 0.074 \\
\hline Shift & $1.94(0.48)$ & $1.79(0.51)$ & $1.98(0.50)$ & $2.12(0.56)$ & $0.019^{\mathrm{E}}$ \\
\hline Emotional control & $1.90(0.43)$ & $1.76(0.42)$ & $1.90(0.42)$ & $2.14(0.56)$ & $0.003^{\mathrm{C}, \mathrm{E}}$ \\
\hline Self-monitor & $1.75(0.47)$ & $1.69(0.53)$ & $1.99(0.48)$ & $2.05(0.61)$ & $0.001^{\mathrm{B}, \mathrm{C}, \mathrm{D}, \mathrm{E}}$ \\
\hline Initiate & $2.00(0.49)$ & $1.91(0.49)$ & $1.94(0.51)$ & $1.89(0.61)$ & 0.484 \\
\hline Working memory & $2.03(0.53)$ & $1.91(0.54)$ & $2.13(0.52)$ & $2.11(0.54)$ & 0.155 \\
\hline Plan/organize & $1.94(0.46)$ & $1.90(0.49)$ & $1.89(0.55)$ & $1.85(0.55)$ & 0.733 \\
\hline Task monitor & $1.93(0.45)$ & $1.90(0.47)$ & $2.11(0.53)$ & $2.04(0.50)$ & 0.097 \\
\hline Organization of materials & $1.88(0.48)$ & $1.88(0.47)$ & $2.00(0.58)$ & $2.06(0.58)$ & 0.161 \\
\hline Behavioral regulation & $7.66(1.45)$ & $7.36(1.50)$ & $8.12(1.57)$ & $8.59(1.87)$ & $0.001^{\mathrm{C}, \mathrm{E}}$ \\
\hline Metacognition & $9.78(1.88)$ & $9.52(1.94)$ & $10.08(2.11)$ & $9.95(2.37)$ & 0.536 \\
\hline \multicolumn{6}{|l|}{ Personality traits } \\
\hline Negative Affectivity & $1.14(0.68)$ & $0.95(0.71)$ & $1.08(0.65)$ & $1.48(0.51)$ & $0.01^{\mathrm{E}}$ \\
\hline Detachment & $1.17(0.62)$ & $1.04(0.63)$ & $1.05(0.60)$ & $1.16(0.61)$ & 0.386 \\
\hline Antagonism & $0.75(0.59)$ & $0.86(0.53)$ & $1.07(0.62)$ & $1.21(0.62)$ & $<0.001^{\mathrm{B}, \mathrm{C}, \mathrm{E}}$ \\
\hline Disinhibition & $1.30(0.70)$ & $1.36(0.59)$ & $1.65(0.72)$ & $1.62(0.58)$ & $0.012^{\mathrm{B}}$ \\
\hline Rigid Perfectionism & $0.75(0.94)$ & $0.77(0.88)$ & $1.18(1.11)$ & $1.19(1.06)$ & 0.019 \\
\hline Psychoticism & $0.92(0.66)$ & $0.85(0.65)$ & $1.15(0.75)$ & $1.28(0.64)$ & $0.013^{\mathrm{C}, \mathrm{E}}$ \\
\hline Identity Integration & $1.26(0.75)$ & $1.16(0.68)$ & $1.46(0.77)$ & $1.52(0.64)$ & 0.074 \\
\hline Relational Capacities & $1.14(0.69)$ & $1.06(0.67)$ & $1.22(0.71)$ & $1.38(0.68)$ & 0.228 \\
\hline
\end{tabular}

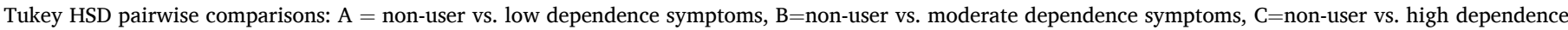

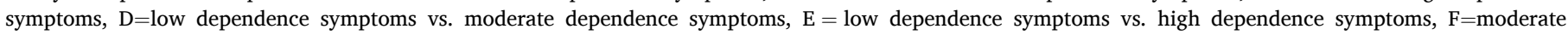
dependence symptoms vs. high dependence symptoms.

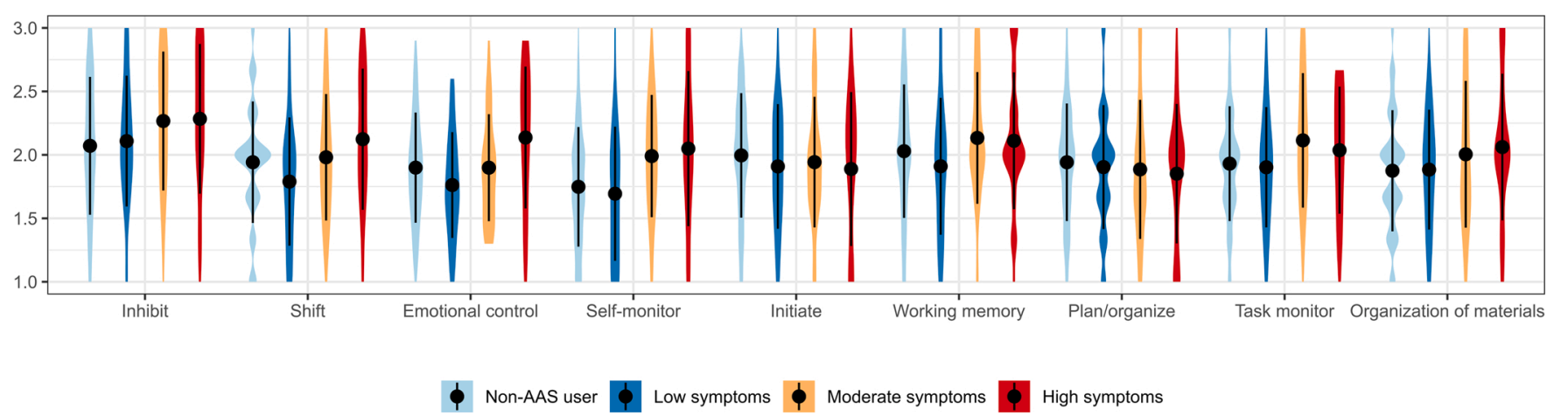

Fig. 2. Distribution of measures of executive function by AAS-using latent class and non-AAS users. Mean and standard deviation in black.

Table 3

Results of multinomial logistic regression; unadjusted OR (95\% CI) with non-AAS users as reference.

\begin{tabular}{|c|c|c|c|c|c|c|}
\hline & Low symptoms & $\mathrm{p}$ & Moderate symptoms & $\mathrm{p}$ & High symptoms & $\mathrm{p}$ \\
\hline \multicolumn{7}{|l|}{ Executive function } \\
\hline Inhibit & $1.13(0.69-1.87)$ & 0.62 & $1.98(1.00-3.92)$ & 0.05 & $2.12(0.98-4.57)$ & 0.06 \\
\hline Shift & $0.53(0.30-0.93)$ & 0.03 & $1.18(0.57-2.43)$ & 0.66 & $2.14(0.94-4.88)$ & 0.07 \\
\hline Self-monitor & $0.79(0.45-1.39)$ & 0.42 & $2.64(1.29-5.38)$ & 0.01 & $3.32(1.49-7.40)$ & 0.00 \\
\hline Initiate & $0.71(0.41-1.21)$ & 0.21 & $0.81(0.40-1.64)$ & 0.55 & $0.65(0.30-1.44)$ & 0.29 \\
\hline Working memory & $0.65(0.39-1.10)$ & 0.11 & $1.46(0.74-2.89)$ & 0.27 & $1.35(0.63-2.88)$ & 0.44 \\
\hline Plan/organize & $0.85(0.48-1.49)$ & 0.57 & $0.78(0.37-1.64)$ & 0.52 & $0.67(0.29-1.55)$ & 0.36 \\
\hline Behavioral regulation & $0.87(0.73-1.05)$ & 0.15 & $1.23(0.97-1.57)$ & 0.09 & $1.53(1.16-2.01)$ & 0.00 \\
\hline Metacognition & $0.93(0.81-1.08)$ & 0.34 & $1.08(0.90-1.30)$ & 0.39 & $1.05(0.85-1.28)$ & 0.66 \\
\hline \multicolumn{7}{|l|}{ Personality traits } \\
\hline Negative Affectivity & $0.65(0.42-1.00)$ & 0.05 & $0.88(0.51-1.53)$ & 0.66 & $2.09(1.13-3.88)$ & 0.02 \\
\hline Detachment & $0.70(0.45-1.11)$ & 0.13 & $0.73(0.40-1.31)$ & 0.29 & $0.97(0.50-1.85)$ & 0.92 \\
\hline Antagonism & $1.40(0.88-2.23)$ & 0.16 & $2.43(1.36-4.33)$ & 0.00 & $3.46(1.79-6.68)$ & 0.00 \\
\hline Disinhibition & $1.16(0.76-1.75)$ & 0.49 & $2.18(1.23-3.85)$ & 0.01 & $2.02(1.05-3.88)$ & 0.03 \\
\hline Rigid Perfectionism & $1.02(0.76-1.37)$ & 0.89 & $1.53(1.08-2.16)$ & 0.02 & $1.55(1.05-2.28)$ & 0.03 \\
\hline
\end{tabular}


psychoticism compared to the low symptoms class and non-AAS users (Table 2, Fig. 3, females included Table S4).

Regression results indicated a negative association with negative affectivity in the low dependence symptoms class (OR: 0.65; 95\% CI $0.42-1.00$ ). Antagonism was positively associated with membership in the moderate and high symptoms classes, with a stronger effect in the high symptoms class (OR: 3.46; 95\% CI: 1.79-6.68). Disinhibition and rigid perfectionism were positively associated with both moderate and high symptoms class membership (Table 3, females included Table S5).

Following backward stepwise variable elimination, the best fitting model included negative affectivity, antagonism, and rigid perfectionism. Negative affectivity was negatively associated with both low (OR 0.42; 95\% CI: 0.25-0.71) and moderate (OR 0.49; 95\% CI: $0.24-0.98$ ) dependence symptoms. Antagonism was positively associated with both moderate and high dependence symptoms, with the stronger effect size for high symptoms (OR 4.04; 95\% CI: 1.82-8.98). Rigid perfectionism was positively associated with moderate symptoms (OR 1.79; 95\% CI 1.16-2.76) (Table S6, females included Tables S7).

\subsection{Correlation of personality traits and EF}

Many personality traits were positively correlated with each other (Figure S1, females included Figure S2). The strongest correlations ( $\geq$ 0.4 ) were seen between the SIPP-118 items, relational capacities and identity integration, and psychoticism, detachment, disinhibition, and negative affectivity. Disinhibition, psychoticism, and identity integration were positively correlated with all EF measures. Conversely, antagonism and rigid perfectionism were only correlated with certain measures, including behavioral regulation, shift, and emotional control, though with a relatively small Kendall's tau $(\leq 0.3)$.

\section{Discussion}

The present study investigated AAS dependence beyond dichotomous measures and identified three classes of AAS dependence symptoms within SUD patients, largely reflecting dependence severity. Despite few differences between the moderate and high symptoms classes on measures of EF and personality, our findings may indicate the course of dependence with continued AAS use, as more symptoms may develop over time with continued use. This approach also allowed us to identify the most severely affected group and ascertain features of EF and personality as potential risk factors. Members of the low symptoms class likely represent SUD patients who used AAS briefly, but did not develop a regimen involving these substances, as reflected by their relatively short duration of use. Additionally, the types of AAS that were most commonly used within each class suggest that the high symptoms group reflects more "typical" AAS use in the weightlifting community with a higher prevalence of injectable AAS, while the compounds used by the low symptoms class suggest that these substances may be used based on availability (Bjørnebekk et al., 2021; Cohen et al., 2007; de
Ronde and Smit, 2020). A large proportion of the moderate symptoms class reported that they took larger amounts of AAS over a longer period of time than originally intended, suggesting that this may be the first symptom of dependence to appear, as has been previously identified in alcohol use disorder (Buu et al., 2012).

While this study cannot establish causality, we identified a putative protective effect of emotional control and shift in the low symptoms class, suggesting that greater functioning in these areas may reduce the risk for developing AAS dependence. Similarly, a putative protective effect was found for negative affectivity, suggesting that those with lower anxiety and hostility may be less prone to severe dependence, as this trait has been identified as a risk factor for SUD and alcohol use disorder (Cavicchioli et al., 2020; McHugh and Kneeland, 2019).

Furthermore, we identified associations between the AAS dependence classes and antagonism, disinhibition, rigid perfectionism, and psychoticism. This is similar to previous studies which have found these characteristics to be associated with AAS use (Cooper et al., 1996; Porcerelli and Sandler, 1995) and SUDs (Arnevik et al., 2019; González et al., 2019; Hasin and Kilcoyne, 2012; Heath et al., 2018), though measures of personality vary. Antagonism was positively associated with both moderate and high dependence symptoms, in increasing strength, suggesting that this trait may represent a risk factor for both initiating AAS use, and for more severe dependence, which was also reflected by the stepwise regression. Negative affectivity likely represents a general risk factor and common characteristic for SUD (Cavicchioli et al., 2020; Heath et al., 2018; McHugh and Kneeland, 2019), which may explain the comparable levels between non-AAS users and those with moderate AAS dependence symptoms in this study. Interestingly, rigid perfectionism is not typically associated with SUD (Moraleda-Barreno et al., 2018), but may be related to attitudes towards doping (Zucchetti et al., 2015). Perfectionism has also been associated with eating disorders (Buzzichelli et al., 2018), particularly with restrictive behaviors (Solomon-Krakus et al., 2020), and exercise addiction (Lichtenstein et al., 2014), suggesting that increased rigid perfectionism may contribute to behaviors that pose a risk to health in an attempt to enhance appearance. Additionally, previous studies have demonstrated a strong relationship between antagonism and disinhibition and cluster B personality disorders, which have been associated with AAS use (Bach et al., 2018; Hauger et al., 2021; Perry et al., 2003).

It is important to note the frequent overlap of personality traits and the possibility of a general factor of personality disorder, which may account for a large portion of comorbidity with SUD (Jahng et al., 2011), as reflected by the correlations in our sample. We found that negative affectivity, detachment, disinhibition, and psychoticism correlated with identity integration and relational capacities, suggesting that these facets may overlap. Both relational capacities and identity integration have been associated with worse treatment outcomes in SUD (Papamalis et al., 2021), indicating that these pathologies may be associated with more severe dependence, as reflected by our findings.

While it is not possible to determine whether deficits in EF or

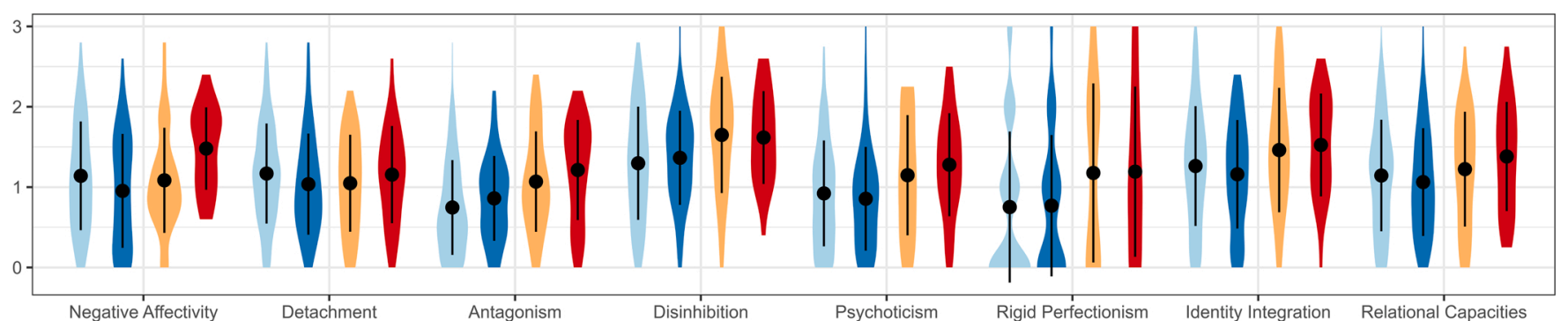

Negative Affectivity Detachment Antagonism Disinhibition Psychoticism Rigid Perfectionism Identity Integration Relational Capacities

Fig. 3. Distribution of personality traits by AAS-using latent class and non-AAS users. Mean and standard deviation in black. 
personality traits are risk factors or consequences of more severe dependency, some features may be present in early life and contribute to risk of SUD development later. For example, there is evidence to suggest some genetic influence on personality traits (Kendler et al., 2019, 2017; Wesseldijk et al., 2018). In addition, previous studies have found that conduct disorder and EF deficits in adolescence may predict SUD in adulthood (Elkins et al., 2007; Wilens et al., 2011). Younger debut age, identified in the moderate and severe symptoms classes in this study, has been associated with both conduct disorder and SUD (Clark et al., 2006; Sung et al., 2004), as well as later addiction severity (Harerimana et al., 2021). Our findings suggest this may also be the case for AAS. Identifying individuals with the most severe dependence symptoms has important implications for both treatment and social interactions, as the traits we found to be present in the high symptoms class have been associated with violent behavior (Lowenstein et al., 2016; Spencer et al., 2019).

It is likely that conduct disorder or antisocial behavior may contribute to use of both psychoactive substances and AAS (Kanayama et al., 2018). Difficulty with self-monitoring, which the results of this study suggest is an important characteristic of more severe AAS dependence, indicates trouble with recognizing the effect of one's social behavior on others. Similarly, AAS dependents have demonstrated reduced ability to infer or interpret the mental state of others compared to non-using weight-lifters (Vaskinn et al., 2020). Furthermore, antagonism and disinhibition may contribute to externalizing behaviors (Chester and West, 2020; Vize et al., 2020) which may put others at risk, including sexual aggression (Kasowski and Anderson, 2020).

In line with previous findings of AAS use among prisoners and SUD patients, AAS use may be a marker for more severe substance use in general (Havnes et al., 2020a). The current findings suggest potential underlying cognitive and personality factors which may explain this relation. Those who use AAS, particularly those that demonstrate signs of severe dependence, appear more likely to have EF deficits and personality pathology, making this group particularly challenging to treat compared to SUD patients who do not use AAS. AAS users in both SUD treatment and the general population report that they do not typically disclose their steroid use, often because they fear judgment, feel that their physician lacks knowledge about AAS, or are simply not asked about AAS use (Bonnecaze et al., 2020; Havnes et al., 2020b). Thus, clinicians should be properly informed about AAS use and the additional risk factors and adverse effects that may occur with using these substances, in order to better understand these patients and provide more appropriate treatment.

\subsection{Limitations}

There are some limitations in the present study. Findings cannot be generalized to all regions, as the study population and their environment, including treatment facilities and resources, are specific to Norway and may vary from other countries. Self-report personality and EF measures may not accurately represent the participant's personality traits or abilities; however the tools used to measure these characteristics are well-regarded within the research community. Reported AAS use may be subject to memory bias, as some users had not used AAS for some time. The questionnaire assessing AAS dependence has not been widely used; however the items are based on the SCID-II, which is a common measure of substance dependence. Furthermore, non-AAS users include those that are in treatment for alcohol use, who may exhibit particular features of personality and EF that differ from those who primarily use illegal substances.

In addition, clustering algorithms including latent class analysis may produce results which lack reliability and validity, particularly when the sample size is relatively small, as in this study (Nylund et al., 2007). Additionally, female AAS users are not well-represented in our study sample and thus our results cannot be generalized to females. It would be valuable to replicate this analysis in additional samples to determine if the classes identified are both generalizable and reliable.

It is important to note that all study participants are in SUD treatment for substances not including AAS, posing a challenge to distinguish the effects of, or risk factors for, AAS use from those of other substances. For example, the high prevalence of stimulant use in the moderate and high dependence groups may explain some of the associations with EF deficits, as use of substances including methamphetamine and cocaine may be associated with cognitive deficits (Jovanovski et al., 2005; Mizoguchi and Yamada, 2019; van der Plas et al., 2009), though this is somewhat disputed (Frazer et al., 2018). Moreover, as participants in the study primarily were in treatment for misuse of classical drugs of abuse it is important to keep in mind that this population of AAS users likely differ substantially from the overall source population of AAS users. Hence, the findings should not be generalized to the overall population of AAS users. Future research should investigate the relationships between EF, personality, and severity of AAS dependence in a population not seeking SUD treatment. Based on the present study, it is also not possible to determine whether these deficits are a result of substance use or a risk factor for SUD. Future studies should utilize longitudinal designs in order to distinguish between risk factors and consequences of AAS use in the SUD population.

\subsection{Conclusions}

Individuals with more severe AAS dependence, comprising individuals that experience a high number of symptoms exceeding the typical cut-off for dependence, likely demonstrate greater problems with behavior regulation and emotional control, compared to SUD patients who do not use AAS. These characteristics represent additional challenges in treatment and should be assessed. Thus, it is important to utilize methods both in clinical practice and future research to identify individuals with more severe dependence challenges in order to provide effective treatment and reduce harm to themselves and others. Equally important is that this information can be used to identify those most at risk of harmful sustained and dependent use in targeted prevention programming. Understanding the risks for more severe dependence, as well as the motivations to continue using AAS despite side effects, will provide more insight into effective health messaging, targeted outreach, appropriate treatment, harm reduction and care pathways. While these findings cannot be generalized to AAS users not in SUD treatment, this population represents a vulnerable section of society. Identifying more severe dependence has clinically relevant implications, as cognitive deficit, personality disorders, and young age are potential risk factors for dropping out of SUD treatment and are thus important variables to take into account (Brorson et al., 2013).

\section{Role of funding source}

Nothing declared.

\section{Contributors}

All authors have contributed substantially to the present work, and all have reviewed and approved the submitted manuscript.

\section{Conflict of Interest}

The authors report no declarations of interest.

\section{Acknowledgments}

This research was funded by grants 2013087 and 2016049 (Dr. Bjørnebekk) from the South-Eastern Norway Regional Health Authority, and by internal research grants from the Division on Mental Health and Addiction (Dr. Bjørnebekk). 
Appendix B. Supporting information

Supplementary data associated with this article can be found in the online version at doi:10.1016/j.drugalcdep.2022.109275.

\section{References}

American Psychiatric Association, 2013. Diagnostic and Statistical Manual of Mental Disorders (DSM-5). American Psychiatric Pub.

Arnevik, E.A., Pedersen, G., Walderhaug, E., Lien, I., Wilberg, T., Hummelen, B., 2019. Measuring personality problems in patients with substance use disorders: a crosssample validation. J. Dual Diagn. 15 (4), 324-332.

Bach, B., Sellbom, M., Skjernov, M., Simonsen, E., 2018. ICD-11 and DSM-5 personality trait domains capture categorical personality disorders: Finding a common ground. Aust. N. Z. J. Psychiatry 52 (5), 425-434.

Bjornebekk, A., Westlye, L.T., Walhovd, K.B., Jorstad, M.L., Sundseth, O.O., Fjell, A.M., 2019. Cognitive performance and structural brain correlates in long-term anabolic androgenic steroid exposed and nonexposed weightlifters. Neuropsychology 33 (4), 547-559.

Bjørnebekk, A., Kaufmann, T., Hauger, L.E., Klonteig, S., Hullstein, I.R., Westlye, L.T., 2021. Long-term anabolic-androgenic steroid use is associated with deviant brain aging. Biol. Psychiatry Cogn. Neurosci. Neuroimaging 6 (5), 579-589.

Blacken, P., Hendriks, V., Pozzi, G., Tempesta, E., Hartgers, C., Koeter, M., Fahrner, E., Gsellhofer, B., Küfner, H., Kokkevi, A., 2010. European Addiction Severity Index EuropASI Manual. European Monitoring Centre for Drugs and Drug Addiction, Lisbon.

Bonnecaze, A.K., O'Connor, T., Aloi, J.A., 2020. Characteristics and Attitudes of Men Using Anabolic Androgenic Steroids (AAS): A Survey of 2385 Men. Am. J. Men. 'S. Health 14 (6), $1557988320966536,1557988320966536$.

Brorson, H.H., Ajo Arnevik, E., Rand-Hendriksen, K., Duckert, F., 2013. Drop-out from addiction treatment: a systematic review of risk factors. Clin. Psychol. Rev. 33 (8), 1010-1024.

Buu, A., Wang, W., Schroder, S.A., Kalaida, N.L., Puttler, L.I., Zucker, R.A., 2012. Developmental emergence of alcohol use disorder symptoms and their potential as early indicators for progression to alcohol dependence in a high risk sample: a longitudinal study from childhood to early adulthood. J. Abnorm Psychol. 121 (4), 897-908.

Buzzichelli, S., Marzola, E., Amianto, F., Fassino, S., Abbate-Daga, G., 2018. Perfectionism and cognitive rigidity in anorexia nervosa: Is there an association? Eur. Eat. Disord. Rev. 26 (4), 360-366.

Cavicchioli, M., Ramella, P., Movalli, M., Prudenziati, F., Vassena, G., Simone, G., Maffei, C., 2020. DSM-5 maladaptive personality domains among treatment-seeking individuals with alcohol use disorder: the role of disinhibition and negative affectivity. Subst. Use Misuse 55 (11), 1746-1758.

Chegeni, R., Pallesen, S., McVeigh, J., Sagoe, D., 2021. Anabolic-androgenic steroid administration increases self-reported aggression in healthy males: a systematic review and meta-analysis of experimental studies. Psychopharmacology 238, 1911-1922.

Chester, D.S., West, S.J., 2020. Trait aggression is primarily a facet of antagonism: evidence from dominance, latent correlational, and item-level analyses. J. Res Pers. $89,89$.

Clark, D.B., Jones, B.L., Wood, D.S., Cornelius, J.R., 2006. Substance use disorder trajectory classes: diachronic integration of onset age, severity, and course. Addict. Behav. 31 (6), 995-1009.

Cohen, J., Collins, R., Darkes, J., Gwartney, D., 2007. A league of their own: demographics, motivations and patterns of use of 1,955 male adult non-medical anabolic steroid users in the United States. J. Int. Soc. Sports Nutr. 4 (1), 12.

Cooper, C.J., Noakes, T.D., Dunne, T., Lambert, M.I., Rochford, K., 1996. A high prevalence of abnormal personality traits in chronic users of anabolic-androgenic steroids. Br. J. Sports Med 30 (3), 246-250.

de Ronde, W., Smit, D.L., 2020. Anabolic androgenic steroid abuse in young males. Endocr. Connect. 9 (4), R102-R111.

Diamond, A., 2013. Executive functions. Annu. Rev. Psychol. 64 (1), 135-168.

Dodge, T., Hoagland, M.F., 2011. The use of anabolic androgenic steroids and polypharmacy: a review of the literature. Drug Alcohol Depend. 114 (2-3), 100-109.

Elkins, I.J., McGue, M., Iacono, W.G., 2007. Prospective effects of attention-deficit/ hyperactivity disorder, conduct disorder, and sex on adolescent substance use and abuse. Arch. Gen. Psychiatry 64 (10), 1145-1152.

Frazer, K.M., Manly, J.J., Downey, G., Hart, C.L.J.J.O.C., 2018. Assessing cognitive functioning in individuals with cocaine use disorder. Neuropsychol., e 40 (6), 619-632.

Garcia-Argibay, M., 2019. The relationship between the big five personality traits, impulsivity, and anabolic steroid use. Subst. Use Misuse 54 (2), 236-246.

González, E., Arias, F., Szerman, N., Vega, P., Mesias, B., Basurte, I., 2019. Coexistence between personality disorders and substance use disorder. Madr. Study Preval. Dual Pathol. Actas Esp. De. Psiquiatr. 47 (6), 218-228.

Griffiths, S., Jacka, B., Degenhardt, L., Murray, S.B., Larance, B., 2018. Physical appearance concerns are uniquely associated with the severity of steroid dependence and depression in anabolic-androgenic steroid users. Drug Alcohol Rev. 37 (5), 664-670.

Hagen, E., Erga, A.H., Hagen, K.P., Nesvåg, S.M., McKay, J.R., Lundervold, A.J., Walderhaug, E., 2016. Assessment of executive function in patients with substance use disorder: a comparison of inventory- and performance-based assessment. J. Subst. Abus. Treat. 66, 1-8.
Harerimana, B., Kerr, M., Csiernik, R., Ng, L.C., Rutembesa, E., Forchuk, C., 2021. Predicting the contribution of age at first substance use and post-traumatic stress disorder to later addiction severity in a clinical sample from sub-saharan africa: implications for prevention and treatment. Int. J. Ment. Health Addict.

Hasin, D., Kilcoyne, B., 2012. Comorbidity of psychiatric and substance use disorders in the United States: current issues and findings from the NESARC. Curr. Opin. Psychiatry 25 (3), 165-171.

Hauger, L.E., Havnes, I.A., Jørstad, M.L., Bjørnebekk, A., 2021. Anabolic androgenic steroids, antisocial personality traits, aggression and violence. Drug Alcohol Depend. 221, 108604.

Hauger, L.E., Westlye, L.T., Bjørnebekk, A., 2020. Anabolic androgenic steroid dependence is associated with executive dysfunction. Drug Alcohol Depend. 208, 107874.

Havnes, I.A., Bukten, A., Rognli, E.B., Muller, A.E., 2020a. Use of anabolic-androgenic steroids and other substances prior to and during imprisonment - Results from the Norwegian Offender Mental Health and Addiction (NorMA) study. Drug Alcohol Depend. 217, 108255.

Havnes, I.A., Jørstad, M.L., McVeigh, J., Van Hout, M.C., Bjørnebekk, A., 2020b. The anabolic androgenic steroid treatment gap: a national study of substance use disorder treatment. Subst. Abus.: Res. Treat. 14, 1178221820904150.

Heath, L.M., Drvaric, L., Hendershot, C.S., Quilty, L.C., Bagby, R.M., 2018. Normative and maladaptive personality trait models of mood, psychotic, and substance use disorders. J. Psychopathol. Behav. Assess. 40 (4), 606-613.

Heffernan, T., Battersby, L., Bishop, P., O’Neill, T.J.T.O.P.J., 2015. Everyday memory deficits associated with anabolic-androgenic steroid use in regular gymnasium users. Open Psychiatry J. 9, 1-6.

Horwitz, H., Andersen, J.T., Dalhoff, K.P., 2019. Health consequences of androgenic anabolic steroid use. J. Intern. Med. 285 (3), 333-340.

Ip, E.J., Lu, D.H., Barnett, M.J., Tenerowicz, M.J., Vo, J.C., Perry, P.J.J.P.T.Jo.H.P., Therapy, D., 2012. Psychological and physical impact of anabolic-androgenic steroid dependence. Psychol. Phys. Impact Anab. Steroid Depend. 32 (10), 910-919.

Jahng, S., Trull, T.J., Wood, P.K., Tragesser, S.L., Tomko, R., Grant, J.D., Bucholz, K.K., Sher, K.J., 2011. Distinguishing general and specific personality disorder features and implications for substance dependence comorbidity. J. Abnorm Psychol. 120 (3), 656-669.

Jovanovski, D., Erb, S., Zakzanis, K.K., 2005. Neurocognitive deficits in cocaine users: a quantitative review of the evidence. J. Clin. Exp. Neuropsychol. 27 (2), 189-204.

Kanayama, G., Brower, K.J., Wood, R.I., Hudson, J.I., Pope Jr., H.G., 2009a. Anabolicandrogenic steroid dependence: an emerging disorder. Addiction 104 (12), 1966-1978.

Kanayama, G., Hudson, J.I., Pope Jr., H.G., 2009b. Features of men with anabolicandrogenic steroid dependence: A comparison with nondependent AAS users and with AAS nonusers. Drug Alcohol Depend. 102 (1-3), 130-137.

Kanayama, G., Hudson, J.I., Pope Jr., H.G., 2010. Illicit anabolic-androgenic steroid use. Horm. Behav. 58 (1), 111-121.

Kanayama, G., Kean, J., Hudson, J.I., Pope Jr., H.G., 2013. Cognitive deficits in long-term anabolic-androgenic steroid users. Drug Alcohol Depend. 130 (1-3), 208-214.

Kanayama, G., Pope, H.G., Hudson, J.I., 2018. Associations of anabolic-androgenic steroid use with other behavioral disorders: an analysis using directed acyclic graphs. Psychol. Med 48 (15), 2601-2608.

Kasowski, A.E., Anderson, J.L., 2020. The association between sexually aggressive cognitions and pathological personality traits in men. Violence Women 26 (12-13), $1636-1655$.

Kendler, K.S., Aggen, S.H., Gillespie, N., Krueger, R.F., Czajkowski, N., Ystrom, E., Reichborn-Kjennerud, T., 2019. The structure of genetic and environmental influences on normative personality, abnormal personality traits, and personality disorder symptoms. Psychol. Med 49 (8), 1392-1399.

Kendler, K.S., Aggen, S.H., Gillespie, N., Neale, M.C., Knudsen, G.P., Krueger, R.F., Czajkowski, N., Ystrom, E., Reichborn-Kjennerud, T., 2017. The genetic and environmental sources of resemblance between normative personality and personality disorder traits. J. Pers. Disord. 31 (2), 193-207.

Kotov, R., Gamez, W., Schmidt, F., Watson, D., 2010. Linking "big" personality traits to anxiety, depressive, and substance use disorders: a meta-analysis. Psychol. Bull. 136 (5), 768-821.

Krueger, R.F., Derringer, J., Markon, K.E., Watson, D., Skodol, A.E.J.Pm, 2012. Initial construction of a maladaptive personality trait model and inventory for DSM-5. Psychol. Med. 42 (9), 1879.

Lichtenstein, M.B., Christiansen, E., Elklit, A., Bilenberg, N., Støving, R.K., 2014. Exercise addiction: a study of eating disorder symptoms, quality of life, personality traits and attachment styles. Psychiatry Res 215 (2), 410-416.

Linzer, D.A., Lewis, J.B., 2011. poLCA: an R package for polytomous variable latent class analysis. J. Stat. Softw, 42, 1-29.

Lowenstein, J., Purvis, C., Rose, K., 2016. A systematic review on the relationship between antisocial, borderline and narcissistic personality disorder diagnostic traits and risk of violence to others in a clinical and forensic sample. Bord. Pers. Disord. Emot. Dysregul 3 (1), 14.

McHugh, R.K., Kneeland, E.T., 2019. Affective vulnerability in substance use disorders. Curr. Opin. Psychol. 30, 54-58.

Mizoguchi, H., Yamada, K., 2019. Methamphetamine use causes cognitive impairment and altered decision-making. Neurochem. Int. 124, 106-113.

Moraleda-Barreno, E., Diaz-Batanero, C., Perez-Moreno, P.J., Gomez-Bujedo, J., Lozano, O.M., 2018. Relations between facets and personality domains with impulsivity: New evidence using the DSM-5 Section III framework in patients with substance use disorders. Pers. Disord. 9 (5), 490-495. 
Nylund, K.L., Asparouhov, T., Muthén, B.O.J.SemAm.J., 2007. Deciding on the number of classes in latent class analysis and growth mixture modeling: A Monte Carlo simulation study. Struct. Eq. Model. Mult. J. 14 (4), 535-569.

Papamalis, F.E., Dritsas, I., Knight, K., 2021. The role of personality functioning on early drop out in outpatient substance misuse treatment. Subst. Use Misuse 56 (8), 1119-1136.

Perry, P.J., Kutscher, E.C., Lund, B.C., Yates, W.R., Holman, T.L., Demers, L., 2003. Measures of aggression and mood changes in male weightlifters with and without androgenic anabolic steroid use. J. Forensic Sci. 48 (3), 646-651.

Piacentino, D., Kotzalidis, G.D., Del Casale, A., Aromatario, M.R., Pomara, C., Girardi, P. Sani, G., 2015. Anabolic-androgenic steroid use and psychopathology in athletes. A systematic review. Curr. Neuropharmacol. 13 (1), 101-121.

Pope Jr., H.G., Wood, R.I., Rogol, A., Nyberg, F., Bowers, L., Bhasin, S., 2014. Adverse health consequences of performance-enhancing drugs: an Endocrine Society scientific statement. Endocr. Rev. 35 (3), 341-375.

Porcerelli, J.H., Sandler, B.A., 1995. Narcissism and empathy in steroid users. Am. J. Psychiatry 152 (11), 1672-1674.

R Core Team, 2018, R: A Language and Environment for Statistical Computing. R Foundation for Statistical Computing, Vienna, Austria.

Roth, R.M., Gioia, G.A., 2005. Behavior rating inventory of executive function-adult version. Psychological Assessment Resources, Lutz, FL.

Sagoe, D., McVeigh, J., Bjørnebekk, A., Essilfie, M.S., Andreassen, C.S., Pallesen, S., 2015. Polypharmacy among anabolic-androgenic steroid users: a descriptive metasynthesis. Subst. Abus. Treat., Prev., Policy 10, 12.

Salinas, M., Floodgate, W., Ralphs, R., 2019. Polydrug use and polydrug markets amongst image and performance enhancing drug users: Implications for harm reduction interventions and drug policy. Int. J. Drug Policy 67, 43-51.

Snow, M.B., Balling, C., Zimmerman, M., 2020. Re-examining borderline personality disorder and substance use disorder: The role of emotion dysregulation. Ann. Clin. Psychiatry.: Off. J. Am. Acad. Clin. Psychiatr. 32 (3), 170-175.

Solomon-Krakus, S., Uliaszek, A.A., Bagby, R.M., 2020. Evaluating the associations between personality psychopathology and heterogeneous eating disorder behaviors: A dimensional approach. Pers. Disord. 11 (4), 249-259.

Spencer, C., Mallory, A.B., Cafferky, B.M., Kimmes, J.G., Beck, A.R., Stith, S.M., 2019. Mental health factors and intimate partner violence perpetration and victimization: A meta-analysis. Psychol. Violence 9 (1), 1-17.

Sung, M., Erkanli, A., Angold, A., Costello, E.J., 2004. Effects of age at first substance use and psychiatric comorbidity on the development of substance use disorders. Drug Alcohol Depend. 75 (3), 287-299.
Tein, J.-Y., Coxe, S., Cham, H., 2013. Statistical power to detect the correct number of classes in latent profile analysis. Struct. Equ. Model.: A Multidiscip. J. 20 (4), 640-657.

Trull, T.J., Freeman, L.K., Vebares, T.J., Choate, A.M., Helle, A.C., Wycoff, A.M., 2018. Borderline personality disorder and substance use disorders: an updated review. Bord. Pers. Disord. Emot. Dysregul 5, 15, 15-15.

van Amsterdam, J., Opperhuizen, A., Hartgens, F., 2010. Adverse health effects of anabolic-androgenic steroids. Regul. Toxicol. Pharm. 57 (1), 117-123.

van der Plas, E.A.A., Crone, E.A., van den Wildenberg, W.P.M., Tranel, D., Bechara, A., 2009. Executive control deficits in substance-dependent individuals: a comparison of alcohol, cocaine, and methamphetamine and of men and women. J. Clin. Exp. Neuropsychol. 31 (6), 706-719.

Vaskinn, A., Hauger, L.E., Bjørnebekk, A., 2020. Theory of mind in users of anabolic androgenic steroids. Psychopharmacol. (Berl. ) 237 (10), 3191-3199.

Verheul, R., Andrea, H., Berghout, C.C., Dolan, C., Busschbach, J.J., van der Kroft, P.J., Bateman, A.W., Fonagy, P.J.P.A., 2008. Severity Indices of Personality Problems (SIPP-118): Development, factor structure, reliability, and validity. Psychol. Assess. 20 (1), 23-34.

Vize, C.E., Collison, K.L., Lynam, D.R., 2020. The importance of antagonism: explaining similarities and differences in psychopathy and narcissism's relations with aggression and externalizing outcomes. J. Pers. Disord. 34 (6), 842-854.

Wesseldijk, L.W., Bartels, M., Vink, J.M., van Beijsterveldt, C.E.M., Ligthart, L., Boomsma, D.I., Middeldorp, C.M., 2018. Genetic and environmental influences on conduct and antisocial personality problems in childhood, adolescence, and adulthood. Eur. Child Adolesc. Psychiatry 27 (9), 1123-1132.

Wilens, T.E., Carrellas, N.W., Martelon, M., Yule, A.M., Fried, R., Anselmo, R., McCabe, S.E., 2017. Neuropsychological functioning in college students who misuse prescription stimulants. Am. J. Addict. 26 (4), 379-387.

Wilens, T.E., Martelon, M., Fried, R., Petty, C., Bateman, C., Biederman, J., 2011. Do executive function deficits predict later substance use disorders among adolescents and young adults? J. Am. Acad. Child Adolesc. Psychiatry 50 (2), 141-149.

World Health Organization, 2019. ICD-11: International Classification of Diseases 11th Revision: the Global Standard for Diagnostic Health Information. World Health Organization.

Zucchetti, G., Candela, F., Villosio, C., 2015. Psychological and social correlates of doping attitudes among Italian athletes. Int. J. Drug Policy 26 (2), 162-168. 